\title{
Aspiration and severe exacerbations in COPD: a prospective study
}

\author{
Lydia Cvejic ${ }^{1,2,3}$, Nadine Guiney ${ }^{1}$, Tiffany Nicholson ${ }^{4}$, Kenneth K. Lau ${ }^{2,4}$, \\ Paul Finlay ${ }^{1}$, Kais Hamza ${ }^{5}$, Christian Osadnik (i) ${ }^{1,3}$, Paul Leong ${ }^{1,2,3}$, \\ Martin MacDonald ${ }^{1,2,3}$, Paul T. King ${ }^{1,2,3}$ and Philip G. Bardin ${ }^{1,2,3}$
}

Affiliations: ${ }^{1}$ Monash Lung and Sleep, Monash Health, Melbourne, Australia. ${ }^{2}$ School of Clinical Sciences, Monash University, Melbourne, Australia. ${ }^{3}$ Hudson Institute and Monash University, Melbourne, Australia. ${ }^{4}$ Diagnostic Imaging, Monash Health, Melbourne, Australia. ${ }^{5}$ School of Mathematical Sciences, Monash University, Melbourne, Australia.

Correspondence: Philip G. Bardin, Monash Lung and Sleep, Monash Hospital and University, 246 Clayton Road, Clayton 3168, Melbourne, Australia. E-mail: philip.bardindmonash.edu

\section{ABSTRACT}

Rationale: Swallow may be compromised in COPD leading to aspiration and adverse respiratory consequences. However, prevalence and consequences of detectable aspiration in stable COPD are not known.

Objectives: We tested the hypothesis that a significant number of patients with stable COPD will have detectable aspiration during swallow (prandial aspiration) and that they would experience more frequent severe acute exacerbations of COPD (AECOPD) over the subsequent 12 months.

Methods: Patients $(n=151)$ with verified and stable COPD of all severities were recruited at a tertiary care hospital. Videofluoroscopy was conducted to evaluate aspiration using Rosenbek's scale for penetration-aspiration during 100-mL cup drinking. AECOPD was documented as moderate (antibiotics and/or corticosteroid treatment) or severe (emergency department admission or hospitalisation) over the ensuing 12 months.

Measurements and main results: Aspiration was observed in 30 out of 151 patients $(19.9 \%, 18$ males, 12 females; mean age 72.4 years). Patients with aspiration had more overall AECOPD events (3.03 versus 2 per patient; $\mathrm{p}=0.022$ ) and severe AECOPD episodes ( 0.87 versus $0.39 ; \mathrm{p}=0.032)$. Severe AECOPD occurred in more patients with aspiration (50\% of patients versus $18.2 \%$; OR 4.5 , CI 1.9-10.5; $\mathrm{p}=0.001$ ) and with silent aspiration ( $36.7 \%$ versus $18.2 \%$; OR 2.6 , CI $1.1-6.2 ; \mathrm{p}=0.045)$. Aspiration was related to a shorter exacerbation-free period during the 12 -month follow-up period $(\mathrm{p}=0.038)$.

Conclusions: Prandial aspiration is detectable in a subset of patients with COPD and was predictive of subsequent severe AECOPD. Studies to examine if the association is causal are essential to direct strategies aimed at prevention of aspiration and AECOPD.

@ERSpublications

This study demonstrates that prandial aspiration occurs in $\sim 20 \%$ of patients with stable COPD and portends severe COPD exacerbations over the next 12 months https://bit.ly/2Tx5btj

Cite this article as: Cvejic L, Guiney N, Nicholson T, et al. Aspiration and severe exacerbations in COPD: a prospective study. ERJ Open Res 2021; 7: 00735-2020 [https://doi.org/10.1183/ 23120541.00735-2020].

This article has supplementary material available from openres.ersjournals.com.

This study is registered at www.anzctr.org.au with identifier number ACTRN12620000513910. All individual de-identified participant data (including data dictionaries) will be shared. Related documents will be available including study protocol and statistical analysis plan. Data are immediately available for a period of 7 years to other researchers with an interest in COPD or related areas.

Received: 12 Oct 2020 | Accepted: 19 Oct 2020

Copyright $\odot$ ERS 2021. This article is open access and distributed under the terms of the Creative Commons Attribution Non-Commercial Licence 4.0. 


\section{Introduction}

COPD may impair airway protection during swallow leading to adverse respiratory outcomes. Factors such as altered laryngopharyngeal musculature and sensitivity, tachypnoea, hyperinflation, hypoxia, gastro-oesophageal reflux, pharmaceutical agents and cigarette smoking may predispose patients with COPD to aspiration [1]. However, it is not clear how often aspiration occurs in stable disease and whether aspiration may predispose to recurrent acute exacerbations of COPD (AECOPD).

Prandial aspiration refers to aspiration that occurs during swallow, as distinct from retrograde aspiration (associated with reflux), microaspiration (involving small amounts of oropharyngeal or gastro-oesophageal contents) or silent aspiration (absence of cough despite material present below the vocal folds) [2]. Aspiration associated with swallow is particularly important due to its associated increased risk of pneumonia [3, 4], yet investigations into the condition in patients with COPD are rare or describe swallowing dysfunction of a different nature [5-10]. Limited data from small studies involving an array of methodologies to detect prandial aspiration suggest the condition may occur in up to $25 \%$ of patients with stable COPD [5, 6]. Prevalence of aspiration in COPD and the relationship between aspiration and exacerbations are not known and warrants investigation.

We hypothesised that a significant number of patients with stable COPD will have detectable prandial aspiration related to more frequent severe AECOPD. State-of-the-art videofluoroscopy was used to detect prandial aspiration in patients with stable COPD, and AECOPD events were documented over the subsequent 12 months.

\section{Methods}

Study design, patients, baseline and follow-up study measurements

A prospective observational cohort study was conducted, and all patients provided written informed consent. The study protocol was approved by the Human Research Ethics Committee of Monash Health, Melbourne, Australia. STROBE (Strengthening the Reporting of Observational Studies in Epidemiology) reporting guidelines were used, and the study is registered with the Australian New Zealand Clinical Trials Registry (ACTRN12620000513910).

Studies were conducted at Monash Lung and Sleep at Monash Medical Centre, a tertiary care hospital in Melbourne, Australia. Community-dwelling patients were identified from a hospital pulmonary function database $\left(\geqslant 10\right.$ pack-year history of smoking, post-bronchodilator forced expiratory volume in $1 \mathrm{~s}\left(\mathrm{FEV}_{1}\right) /$ forced vital capacity (FVC) $\leqslant 0.7$ and $\mathrm{FEV}_{1}<80 \%$ predicted [11]) and invited to participate. They had to have a diagnosis of COPD by a general practitioner or respiratory physician, stable lung disease in the preceding 12 weeks and had to be aged 40-80 years. Exclusions are noted in the supplementary material.

The Airways Questionnaire 20 (AQ20), a short version of the St George's Respiratory Questionnaire (SGRQ) [12], was used to evaluate quality of life. The Eating Assessment Tool-10 (EAT-10) [13] identifies abnormal swallowing symptoms (score of $\geqslant 3$ ). The Oral Health Assessment Tool (OHAT) was administered to identify oral health issues [14].

Measurements of spirometry and other outcomes are detailed in the supplementary material.

\section{Videofluoroscopy}

Dynamic fluoroscopic imaging used the Philips MultiDiagnost Eleva with Flat Detector unit (Eleva, Philips Healthcare, Amsterdam, Netherlands) to record images at 30 frames $\cdot \mathrm{s}^{-1}$. Total radiation dose for each patient was $<0.3$ millisieverts. Images were archived in de-identified format. During videofluoroscopy patients were positioned in the seated position. Images were acquired in lateral and oblique positions. Standardised thin oral liquid barium contrast solution $(100 \mathrm{~mL})$ at room temperature at $22 \%$ weight-to-volume barium concentration [15] was prepared from the X-Opaque-HD barium powder (MCI, Melbourne, Australia) combined with thin fruit juice. Liquid barium was self-administered by each patient during videofluoroscopy. It is possible that rapid drinking predisposes to aspiration, and therefore two methods of ingestion (normal drinking at ease and rapid drinking) were evaluated. Patients were allocated in random fashion to either usual cup drinking, then rapid drinking or the reverse. Instructions were to: "swallow as you normally would" and then, after a 1-min recovery interval, "swallow as quickly as possible". The recovery interval was designed to allow time for clearance of potential pharyngo-oesophageal residue. The penetration-aspiration scale (PAS) was used to quantify the presence of penetration-aspiration as validated by Rosenbek [2]. No or momentary penetration of contrast material was scored as $1-2$. Unsafe penetration was defined as scores of $3-5$, aspiration was scored as $6-8$, with silent aspiration (absence of cough) scored as 8. All fluoroscopy data were stored and then randomly analysed at the completion of the 12-month follow-up period. Two independent certified speech pathologists blinded to the study generated the PAS scores. PAS scoring was judged at conclusion of video 
time frame for individual swallow tasks. The highest score for the two swallowing methods was used for analyses. Evaluation of images was done using pause, frame-by-frame, slow motion and reverse options. Intra-observer repeatability (kappa) of observation was $>95 \%$ based on $15 \%$ of randomly selected studies $(n=23)$. If there was discrepancy in penetration-aspiration score between observers, agreement was reached by consensus.

\section{Assessment of AECOPD over 12 months}

Episodes of AECOPD in the year prior to study were obtained by patient recall. AECOPD episodes during the 12 months of study were identified using in-person 3-monthly telephone interviews and methodology as detailed by Bischoff and co-workers [16]. All episodes were verified by examination of medical records. Attempts were not made to identify mild AECOPD (worsening of COPD symptoms only) with no healthcare intervention. Moderate AECOPD was defined as a history of worsened COPD symptoms requiring treatment with antibiotics and/or systemic corticosteroids by a general practitioner without emergency department (ED) review or hospital admission. Severe AECOPD was defined as worsening of COPD symptoms that culminated in ED admission with or without hospitalisation for AECOPD [11]. Frequent exacerbators were characterised as patients having $\geqslant 2$ exacerbations per year of any severity [17].

\section{Statistical analysis}

Primary outcomes were the proportion of patients with detectable aspiration, total number of AECOPD events and patients with at least one episode of severe AECOPD. Secondary outcomes were moderate and combined moderate-severe AECOPD events, and changes from baseline in lung function, exhaled nitric oxide fraction $\left(F_{\mathrm{ENO}}\right)$, and AQ20 and EAT-10 scores. Sample size was based on an estimated prevalence of aspiration of $25 \%$ in COPD [6]. We assumed that the number of patients with aspiration and severe

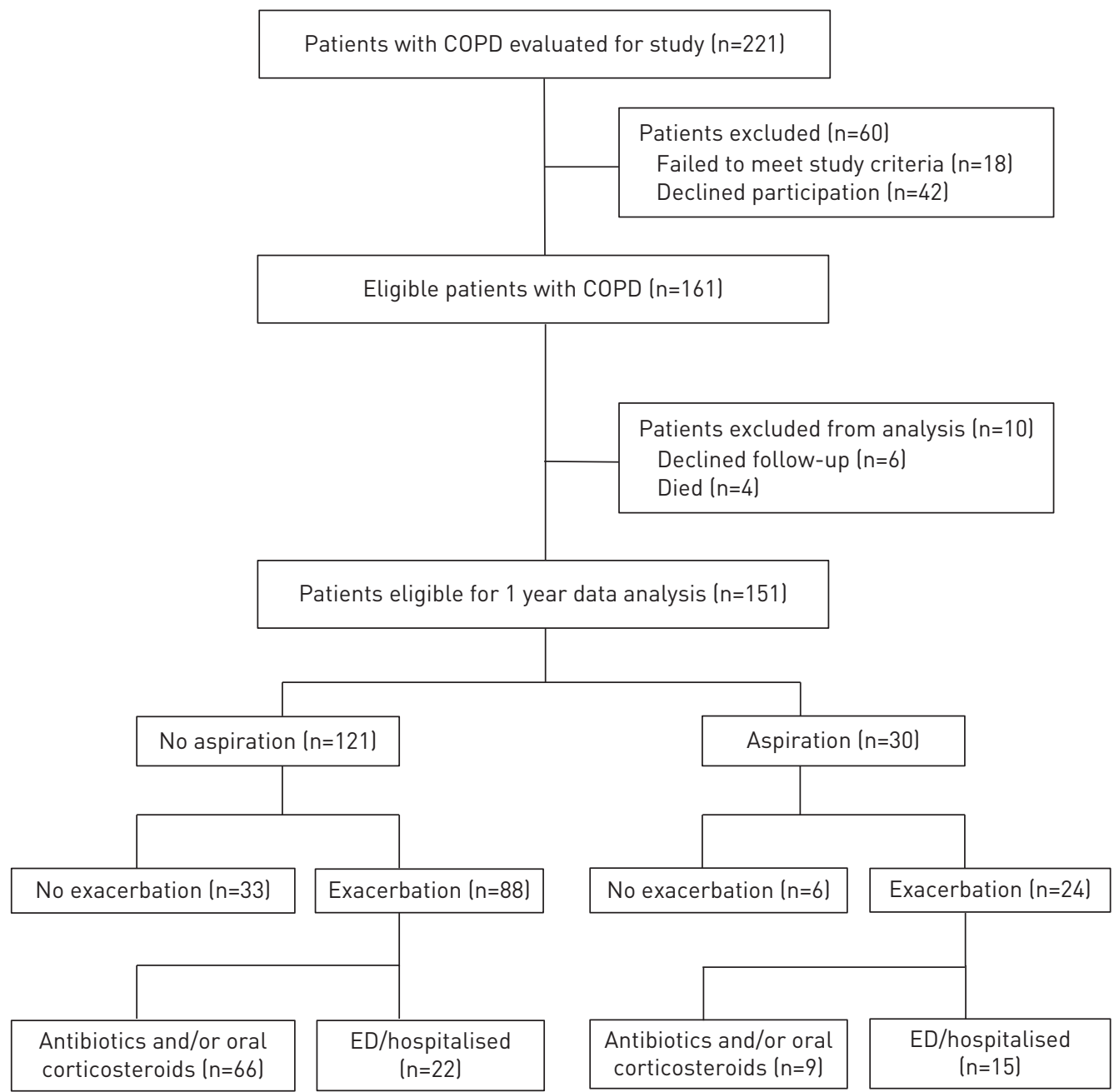

FIGURE 1 Consort diagram of patient participation in the study. ED: emergency department. 
AECOPD would be twice those without aspiration. To achieve statistical power of $80 \%$ with $\mathrm{p} \leqslant 0.05$, the study required 134 patients.

Data were analysed using statistical software package SPSS version 24+. Univariate and multivariate analyses were used to examine whether aspiration could be linked to COPD severity ( $\mathrm{FEV}_{1}$ or $\mathrm{FEV}_{1} / \mathrm{FVC}$ ratio), body mass index, baseline respiratory rate, dysphonia, comorbidities, long-term oral corticosteroids, sedatives, OHAT scores and EAT-10 scores. Appropriate regression analyses were conducted to identify variables that may confound the association between aspiration and AECOPD events. Factors evaluated were age, sex, body mass index (BMI), $\mathrm{FEV}_{1}, \mathrm{FEV}_{1} / \mathrm{FVC}$ ratio, previous exacerbation history, comorbidities and medications. We calculated 95\% confidence intervals. Survival analysis was conducted using the Kaplan-Meier method with log-rank testing. All reported tests were two-tailed and significance was set at $\mathrm{p} \leqslant 0.05$.

\section{Results}

Patients and aspiration

Overall, 221 patients were screened for inclusion in the study (figure 1) and 60 were excluded (42 declined participation; 18 did not meet entry criteria). The remaining 161 patients entered the study of whom 10

TABLE 1 Baseline characteristics of 151 patients enrolled in studies of aspiration in COPD

\begin{tabular}{|c|c|c|}
\hline & Aspiration not detected & Aspiration ${ }^{\#}$ detected \\
\hline Subjects $\mathrm{n}$ & 121 & 30 \\
\hline Age years (range) & $70.2 \pm 5.1(60.1-80.6)$ & $72.4 \pm 4.3^{9}(65.7-78.8)$ \\
\hline Male/female & $74 / 47$ & $18 / 12$ \\
\hline Body mass index $\mathrm{kg} \cdot \mathrm{m}^{-2}$ & $29.4 \pm 5.8$ & $27.7 \pm 6.4$ \\
\hline FEV $1 \%$ predicted & $49.2 \pm 15.3$ & $45.3 \pm 15.3$ \\
\hline $\mathrm{FEV}_{1} / \mathrm{FVC}$ ratio $\%$ & $53.1 \pm 13.9$ & $51.0 \pm 11.1$ \\
\hline TLC $\%$ predicted & $129.2 \pm 22.0$ & $129.0 \pm 20.5$ \\
\hline RV/TLC \% & $58.4 \pm 8.8$ & $60.5 \pm 8.2$ \\
\hline$F_{\text {ENO }} \mathrm{ppb}$ & $24.5 \pm 23.8$ & $22.4 \pm 22.1$ \\
\hline $\mathrm{S}_{\mathrm{pO}_{2}} \%$ & $95.3 \pm 1.8$ & $94.7 \pm 2.8$ \\
\hline Respiratory rate breaths $\cdot \min ^{-1}$ & $17.9 \pm 4.0$ & $18.7 \pm 4.7$ \\
\hline \multicolumn{3}{|l|}{ Comorbidities } \\
\hline Cardiovascular disease & $99(82)$ & $28(93)$ \\
\hline Chronic kidney disease & $7(6)$ & $1(3)$ \\
\hline Gastro-oesophageal reflux disease & $72(60)$ & 17 (57) \\
\hline Obstructive sleep apnoea & $20(17)$ & $3(10)$ \\
\hline Diabetes & $18(15)$ & 7 (23) \\
\hline Anxiety-depression & $27(22)$ & $8(27)$ \\
\hline \multicolumn{3}{|l|}{ Medication } \\
\hline ICS/LABA only & $15(12)$ & 2 (7) \\
\hline ICS/LABA/LAMA & $94(78)$ & $27(90)$ \\
\hline Systemic corticosteroids (long term) & $36(30)$ & $12(40)$ \\
\hline Antibiotics (long term) & $28(23)$ & $7(23)$ \\
\hline Oxygen therapy & $21(17)$ & $7(23)$ \\
\hline Influenza vaccination & $41(34)$ & $11(37)$ \\
\hline Pneumococcal vaccination & $11(9)$ & $4(13)$ \\
\hline Antihypertensives & $96(79)$ & $26(87)$ \\
\hline Antianxiety/antidepressant & 47 (39) & $13(43)$ \\
\hline Angiotensin-converting enzyme inhibitors & $26(22)$ & $7(23)$ \\
\hline Reflux medications & $79(65)$ & $17(57)$ \\
\hline AQ20 score & $9.6 \pm 4.2$ & $10.9 \pm 3.9$ \\
\hline$A Q 20$ score $>8$ & $79(65)$ & $23(77)$ \\
\hline EAT-10 score & $2.3 \pm 3.9$ & $2.9 \pm 4.6$ \\
\hline OHAT score & $2.2 \pm 2.1$ & $3.1 \pm 2.7$ \\
\hline Resting saliva $\mathrm{pH}$ & $6.5 \pm 0.5$ & $6.4 \pm 0.7$ \\
\hline \multicolumn{3}{|c|}{$\begin{array}{l}\text { Data are presented as mean } \pm \mathrm{SD} \text { or } \mathrm{n}(\%) \text {, unless otherwise indicated. } \mathrm{FEV}_{1} \text { : forced expiratory volume in } \\
1 \mathrm{~s} \text {; FVC: forced vital capacity; TLC: total lung capacity; RV: residual volume; } F_{\mathrm{ENO}} \text { : exhaled nitric oxide } \\
\text { fraction; } \mathrm{S}_{\mathrm{pO}_{2}} \text { : oxygen saturation measured by pulse oximetry; ICS: inhaled corticosteroids; LABA: } \\
\text { long-acting beta agonists; LAMA: long-acting muscarinic antagonist; AQ20: Airways Questionnaire-20; } \\
\text { EAT-10: Eating Assessment Tool; OHAT: Oral Health Assessment Tool. }{ }^{\#} \text { : aspiration score of } 6-8 \text { on the } \\
\text { penetration-aspiration scale [2]; }{ }^{\emptyset:} \mathrm{p} \leqslant 0.02 \text {. }\end{array}$} \\
\hline
\end{tabular}


patients ( 2 with aspiration) failed to complete 12 months of follow-up (6 declined follow-up, 4 died: 2 pneumonia, 1 post-operative complications, 1 bowel obstruction). Characteristics of these 10 patients are included in supplementary table S1. Baseline patient demographic data for 151 patients (mean age $70.6 \pm 5.0$ years; mean $\pm \mathrm{SD}$ ) who completed studies over 12 months are shown in table 1 and figure 1 . Aspiration (PAS scores 6-8) was detected in 30/151 patients (19.9\%). Silent aspiration (PAS score 8) was found in $22 / 151$ patients $(14.6 \%)$ and in the majority of those patients in whom aspiration was detected (22/30 patients, $73.3 \%)$. Penetration plus aspiration (PAS scores 3-8) was detected in 48/151 patients (31.8\%) and penetration only (PAS scores $3-5)$ in 18 patients $(11.9 \%)$.

Patients with aspiration were slightly older $(72.4 \pm 4.3$ versus $70.2 \pm 5.1, \mathrm{p}=0.02$, table 1$)$. Univariate and multivariate analyses found no evidence linking aspiration to COPD severity ( $\mathrm{FEV}_{1}$ or $\mathrm{FEV}_{1} / \mathrm{FVC}$ ratio), body mass index, AQ20 scores, baseline respiratory rate, dysphonia, comorbidities, long-term oral corticosteroids, sedatives, OHAT scores and EAT-10 scores. Interestingly, penetration plus aspiration (PAS scores $>2$ ) were detected more frequently in diabetes mellitus despite the limited number of patients with a history of the condition $(\mathrm{n}=25 ; 14 / 25$ with penetration and aspiration; $\mathrm{p}=0.01)$.

Aspiration occurred in 19/30 patients during normal drinking and in 15/30 patients during rapid drinking, and aspiration was observed in $4 / 30$ patients with both methods. Overall PAS scores were $2.39 \pm 2.12$ for normal drinking and $2.45 \pm 1.93$ for rapid drinking $(\mathrm{p}=0.81)$.

\section{Aspiration and AECOPD}

In the year prior to study, 55 patients (out of $151 ; 36.4 \%$ ) had at least one AECOPD event of any severity. There were prior events in $13 / 30$ patients (43.3\%) with aspiration and 42/121 in the group with no aspiration $(34.7 \% ; \mathrm{p}=0.402)$. The number of patients with at least one severe AECOPD episode in the prior year was $11 / 30(36.7 \%)$ in the aspiration group and $24 / 121(19.8 \%)$ in the no aspiration group $(\mathrm{p}=0.057)$.

All patients could be contacted by phone (occasionally after repeated attempts) after 3, 6, 9 and 12 months to administer the AECOPD questionnaire and all reported AECOPD events were verified by examination of medical records. Overall, 334 AECOPD moderate and severe episodes were recorded in the study group over 12 months of follow-up. There were 91 events recorded in patients with aspiration $(n=30)$ and 243 events in the no aspiration group $(n=121 ; 3.03$ events per patient in the aspiration group versus 2.0 per patient; $\mathrm{p}=0.022$ ). Patients with aspiration had a total of 26 severe AECOPD events noted in 30 patients versus 48 severe events in 121 patients with no aspiration $(0.87$ events per patient versus $0.39 ; \mathrm{p}=0.032$; figure $2 \mathrm{a}$, left panel).

Individually 112 patients experienced at least one episode of AECOPD of any severity over the 12 months of follow-up, 24/30 patients with aspiration (80\%) and 88/121 (72.7\%) if aspiration was absent ( $\mathrm{p}=0.491)$. However, more patients with aspiration had severe AECOPD (15/30; 50\%) versus individuals with no aspiration (22/121 (18.2\%); OR 4.5, CI 1.9-10.5; $\mathrm{p}=0.001$; figure $2 \mathrm{a}$, right panel). Similarly, severe

a)

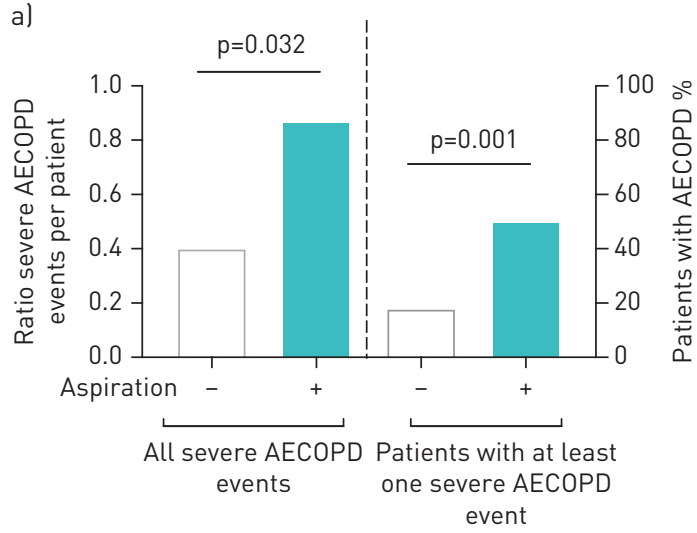

b)

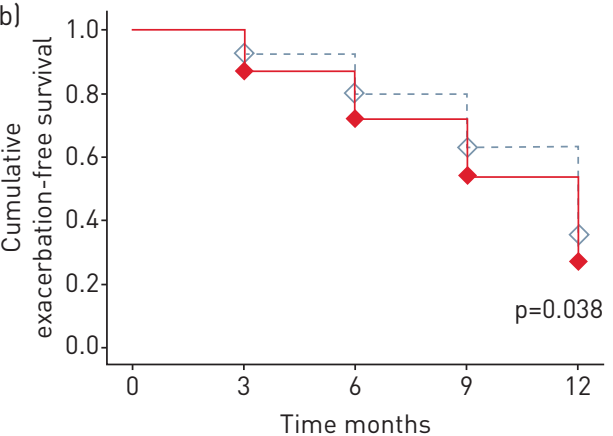

FIGURE 2 a) Aspiration was associated with severe episodes of acute exacerbations of COPD (AECOPD). Left panel: severe AECOPD events were more frequent in patients with aspiration (ratio $0.87 ; n=30$ ) than if no aspiration (ratio $0.39 ; n=121$ ). Right panel: number $(\%)$ of patients with at least one severe episode was greater in patients with aspiration (50\%) than if no aspiration (18\%). (-): aspiration not detected; (+): aspiration detected. b) Kaplan-Meier analysis of patients with no aspiration (open diamonds) and aspiration (closed diamonds) who were exacerbation-free over 12 months of follow-up. Difference between groups analysed using log-rank testing. 
AECOPD was more frequent in patients with silent aspiration (36.7\% versus $18.2 \%$; OR 2.6, CI $1.1-6.2$; $\mathrm{p}=0.045)$. Aspiration was related to a shorter exacerbation-free period in the 12-month follow-up period $(\mathrm{p}=0.038$; figure $2 \mathrm{~b})$.

Appropriate regression analyses were conducted to identify variables that may confound the association between aspiration and AECOPD events. Factors evaluated were age, sex, AQ20 score, BMI, FEV $1, \mathrm{FEV}_{1} /$ FVC ratio, previous exacerbation history, comorbidities and medications. None of these variables altered the association of aspiration with AECOPD. Subgroup analyses of severity and prior history of AECOPD are shown in figure 3 and supplementary table S2.

\section{Pulmonary function and $\mathrm{F}_{E N O}$ measurements}

After 6 months all indices were unchanged between patients with and without aspiration (data not shown). Hyperinflation has been proposed as a factor favouring aspiration [18, 19], but both total lung capacity (TLC) and residual volume (RV)/TLC were not predictive. $F_{\text {ENO }}$ levels $\geqslant 25 \mathrm{ppb}$ were detected in $31 / 151$ patients $(20.5 \%)$ and $\geqslant 50 \mathrm{ppb}$ in $7 / 151(4.6 \%)$, and there was no association with aspiration.

\section{EAT-10 scores and other patient characteristics}

EAT-10 scores $\geqslant 3$ at baseline were noted in $8 / 30$ from the aspiration group (26.7\%) versus $37 / 121$ (30.6\%) if aspiration was absent. EAT-10 scores $>9$ have been proposed as a marker of aspiration [8] but were not predictive (data not shown). Other baseline characteristics including oral health risk measurements and presence of dysphonia $(23 / 151 ; 15.2 \%)$ were not associated with aspiration.

\section{Discussion}

We hypothesised that prandial aspiration occurs in COPD contributing to severe episodes of AECOPD. Our findings establish that aspiration, measured via "gold standard" videofluoroscopy, is found in $20 \%$ of patients and that individuals with evidence of aspiration have an increased propensity to severe AECOPD. Further research is needed to establish whether this association is causative, to define pertinent mechanisms and to investigate practical strategies to diagnose, manage and prevent aspiration in COPD.

Eating and swallowing are important aspects of everyday living. During normal swallow, the larynx serves as a valving mechanism to provide protection from aspiration of liquid or solid material [20]. Laryngeal penetration occurs when there is entry of material into the laryngeal vestibule at or above the true vocal folds that can be cleared by supraglottic and subepiglottic compression [21, 22], expiration [23] or cough. Aspiration is defined as progression of penetrated material below the true vocal folds. Studies in healthy individuals have indicated that prandial aspiration is rare across all age groups [24-26]. However, in COPD penetration and aspiration with swallow may take place more frequently and could be of prognostic significance due to its association with pneumonia [3, 4].

Uncertainty surrounds the prevalence of prandial aspiration in stable COPD. Our previous [6] and other small studies [5] have suggested that aspiration is detectable in $\sim 25 \%$ of stable COPD, and two retrospective studies noted aspiration in up to $40 \%[7,8]$. However, several other investigations failed to detect any evidence of aspiration in this patient group [19, 27-29]. These differences are likely to reflect methodological variations including poorly characterised, small patient study groups, confounding by comorbidities (such as neurological and swallow impairment) and use of small volume or solid contrast materials and high liquid viscosity that may preclude detection of aspiration.

The current study recruited a larger cohort of patients with COPD compared to previous smaller studies $[5,6,8,10,19,27-29]$. Patients had stable, verified disease at baseline, conditions that may predispose to

FIGURE 3 Subgroup analyses of history and types of acute exacerbations of COPD (AECOPD) associated with aspiration or no aspiration. Prior: 12 months prior to study; Current: 12 months of current study.

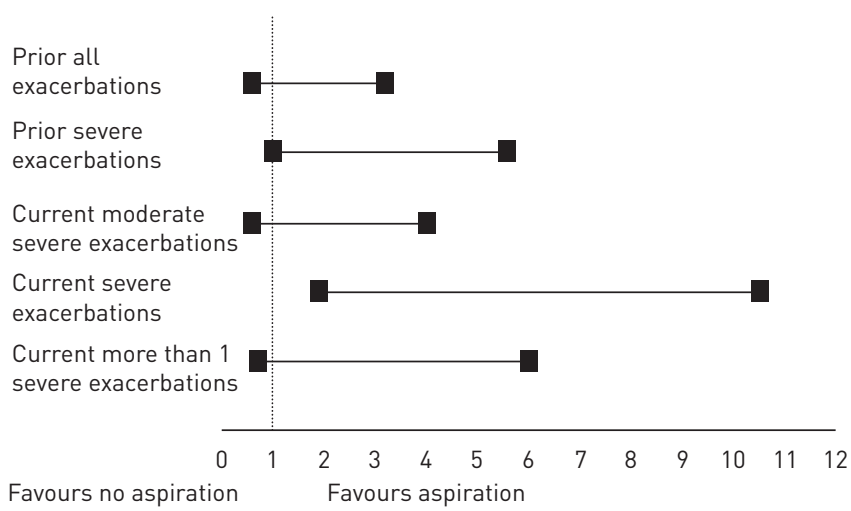


prandial aspiration were excluded and the volume of contrast material was optimised for accurate imaging by means of videofluoroscopy. In this context our findings confirm that aspiration can be detected in up to one fifth of patients with stable COPD, confirming previous small studies $[5,6]$. However, since testing was only performed on one occasion, it is possible that the recorded prevalence of $20 \%$ is an underestimation, and it will also be important to assess in further studies whether aspiration is persistently detectable.

Up to $30 \%$ of AECOPD events have no discernible cause, and other mechanisms such as aspiration may play a role [30-32]. Our previous case-control study hinted at adverse outcomes and more frequent severe AECOPD events in patients who had detectable prandial aspiration [6]. The current study therefore examined whether aspiration is associated with more frequent ED or hospital admission for AECOPD over a 12-month period. The study findings provide affirmative data with increases in overall as well as individual severe AECOPD episodes in patients with aspiration. Importantly, there was a four-fold increase in odds ratio linking aspiration with severe episodes of AECOPD in individual patients indicating that this association was not the result of a few "super-exacerbators". These observations provide evidence that aspiration itself, or as a marker for other predisposing factors such as older age and sarcopenia, is associated with a key adverse outcome in COPD. For that reason, aspiration merits consideration in diagnostic and management approaches aiming to prevent severe AECOPD, perhaps more so in patients who have a history of frequent severe events. Future research examining aspiration and differentiating the causes of AECOPD in detail will help to ascertain the extent to which the association is causal and to explain how aspiration contributes to AECOPD.

Aspiration may cause incremental lung damage and could contribute to the excess decline in lung function noted in COPD [17, 33]. We assessed whether a greater decline in function was measurable 6 months after detection of aspiration (review after 12 months was not feasible due to logistic constraints). No differences in any parameters were noted, a not unexpected result given relatively small patient numbers, individual variations in lung function decline and the short period of study. $F_{\mathrm{ENO}}$, as one measure of airway inflammation, was also evaluated at baseline and after 6 months with no detectable differences.

It would be useful to identify clinical or other parameters predictive of aspiration but in this respect our findings were disappointing. Although patients with aspiration had a higher age than those without aspiration, this finding is of doubtful clinical significance given a difference in mean age of only $\sim 2$ years (table 1). Notably, aspiration was not linked to lower $\mathrm{FEV}_{1}$ measurements or higher lung volumes (TLC) nor was there an association with respiratory rate at rest.

How and why aspiration occurs in COPD is not understood. Our data indicate that reduced laryngopharyngeal sensitivity may be important since the majority of patients had silent aspiration (Rosenbek PAS score 8 noted in $>70 \%$ of individuals with aspiration) implying a degree of airway sensory impairment in this group. Absence of an effective cough reflex may thus reflect a reduced ability to sense aspirated material and to generate appropriate cough and other protective responses to clear the airway. We therefore posit that a dysfunctional "middle airway", perhaps due to reduced timing of laryngeal vestibular closure and sensory mechanisms in COPD [5, 34], may underlie defective protection against aspiration. Finally, an interesting finding was more frequent penetration-aspiration in patients with a history of diabetes mellitus, a condition linked with sarcopenia [35], laryngeal sensory disruption [36], diabetic neuropathy and abnormal oral bacterial loads [37].

The current investigations have several caveats. First, it was a single tertiary centre study with a limited number of patients. Next, an age-matched healthy control group was not studied. Original design of the study had included this group, but the investigators were unable to obtain ethics approval due to local restrictions on radiation exposure for research purposes in healthy individuals. Moreover, there is ample evidence that aspiration is rare in healthy persons [24-26], and comparison of patients with COPD, with and without aspiration, has yielded helpful information. Third, AECOPD events were not assessed during the event itself but documented 3-monthly by patient self-report using a healthcare-based questionnaire combined with medical record confirmation that has been shown to have acceptable accuracy in this context [16]. Fourth, low-dose systemic glucocorticoids $\left(10 \mathrm{mg} \cdot\right.$ day $^{-1}$ or less) were used in $\sim 30 \%$ of patients. Although not recommended by current Global Initiative for Chronic Obstructive Lung Disease (GOLD) guidelines, similar high levels of oral glucocorticoid use have been reported in other countries $[38,39]$. This medication may impact muscle function leading to AECOPD, even though no association with aspiration or AECOPD was detected. Finally, other quantitative assessments such as intranasal pressure measurement for quantification of respiratory phase during swallow $[6,18]$, hand grip strength to assess associations with sarcopenia and a standardised instrument for frailty or age-related susceptibility may have provided additional useful information. 
In conclusion, prandial aspiration can be detected in a subgroup of patients with COPD. The presence of aspiration is associated with severe AECOPD requiring ED or hospital admission. It is unclear why aspiration occurs and how this may predispose to severe episodes of acute deterioration. Future research should aim to verify causative links, improve understanding of mechanistic aspects, examine early and accurate diagnosis and design appropriate studies testing effective approaches to prevent aspiration. Finally, the findings reinforce the importance of swallow-breathing strategies [40] in COPD educational and rehabilitation programmes.

Author contributions: Conception and design: L. Cvejic, P. Finlay, P.T. King and P.G. Bardin; data acquisition: L. Cvejic, N. Guiney and T. Nicholson; data analysis: L. Cvejic, K.K. Lau, K. Hamza and P.G. Bardin; drafted and critically revised the manuscript: L. Cvejic, C. Osadnik, P. Leong, M. MacDonald, P.T. King and P.G. Bardin; final approval for publication: all authors

Conflict of interest: L Cvejic has nothing to disclose. N. Guiney has nothing to disclose. T. Nicholson has nothing to disclose. K.K. Lau has nothing to disclose. P. Finlay has nothing to disclose. K. Hamza has nothing to disclose C. Osadnik reports they were the recipient of a Lung Foundation Australia COPD Research Fellowship (2016-2018; AUD $\$ 160000)$ and is the recipient of a Rebecca L. Cooper Medical Research Foundation Project Grant (2020-2021; AUD\$100000). P. Leong is supported by the National Health and Medical Research Council Postgraduate Scholarship and the Royal Australasian College of Physicians, outside of this work. Views expressed do not necessarily reflect those of the funding bodies. M. MacDonald has nothing to disclose. P.T. King has nothing to disclose. P.G. Bardin has nothing to disclose.

\section{References}

1 Cvejic L, Bardin PG. Swallow and aspiration in chronic obstructive pulmonary disease. Am J Respir Crit Care Med 2018; 198: 1122-1129.

2 Rosenbek JC, Robbins JA, Roecker EB, et al. A penetration-aspiration scale. Dysphagia 1996; 11: 93-98.

3 Almirall J, Rofes L, Serra-Prat M, et al. Oropharyngeal dysphagia is a risk factor for community-acquired pneumonia in the elderly. Eur Respir J 2013; 41: 923-928.

4 Pikus L, Levine MS, Yang YX, et al. Videofluoroscopic studies of swallowing dysfunction and the relative risk of pneumonia. AJR Am J Roentgenol 2003; 180: 1613-1616.

5 Clayton NA, Carnaby GD, Peters MJ, et al. Impaired laryngopharyngeal sensitivity in patients with COPD: the association with swallow function. Int J Speech Lang Pathol 2014; 16: 615-623.

6 Cvejic L, Harding R, Churchward T, et al. Laryngeal penetration and aspiration in individuals with stable COPD. Respirology 2011; 16: 269-275.

7 Good-Fratturelli MD, Curlee RF, Holle JL. Prevalence and nature of dysphagia in VA patients with COPD referred for videofluoroscopic swallow examination. J Commun Disord 2000; 33: 93-110.

8 Regan J, Lawson S, De Aguiar V. The Eating Assessment Tool-10 predicts aspiration in adults with stable chronic obstructive pulmonary disease. Dysphagia 2017; 32: 714-720.

9 Robinson DJ, Jerrard-Dunne P, Greene Z, et al. Oropharyngeal dysphagia in exacerbations of chronic obstructive pulmonary disease. Eur Geriatr Med 2011; 2: 201-203.

10 Garand KL, Strange C, Paoletti L, et al. Oropharyngeal swallow physiology and swallowing-related quality of life in underweight patients with concomitant advanced chronic obstructive pulmonary disease. Int J Chron Obstruct Pulmon Dis 2018; 13: 2663-2671.

11 Vogelmeier CF, Criner GJ, Martinez FJ, et al. Global Strategy for the Diagnosis, Management, and Prevention of Chronic Obstructive Lung Disease 2017 Report. GOLD Executive Summary. Am J Respir Crit Care Med 2017; 195: $557-582$.

12 Jones PW, Quirk FH, Baveystock CM, et al. A self-complete measure of health status for chronic airflow limitation. The St. George's Respiratory Questionnaire. Am Rev Respir Dis 1992; 145: 1321-1327.

13 Belafsky PC, Mouadeb DA, Rees CJ, et al. Validity and reliability of the Eating Assessment Tool (EAT-10). Ann Otol Rhinol Laryngol 2008; 117: 919-924.

14 Chalmers JM, King PL, Spencer AJ, et al. The oral health assessment tool - validity and reliability. Aust Dent J 2005; 50: 191-199.

15 Steele CM, Molfenter SM, Peladeau-Pigeon M, et al. Challenges in preparing contrast media for videofluoroscopy. Dysphagia 2013; 28: 464-467.

16 Bischoff E, Boer L, Molema J, et al. Validity of an automated telephonic system to assess COPD exacerbation rates. Eur Respir J 2012; 39: 1090-1096.

17 Hurst JR, Vestbo J, Anzueto A, et al. Susceptibility to exacerbation in chronic obstructive pulmonary disease. $N$ Engl J Med 2010; 363: 1128-1138.

18 Gross RD, Atwood CW Jr, Ross SB, et al. The coordination of breathing and swallowing in chronic obstructive pulmonary disease. Am J Respir Crit Care Med 2009; 179: 559-565.

19 Mokhlesi B, Logemann JA, Rademaker AW, et al. Oropharyngeal deglutition in stable COPD. Chest 2002; 121: 361-369.

20 Ludlow CL. Laryngeal reflexes: physiology, technique, and clinical use. J Clin Neurophysiol 2015; 32: 284-293.

21 Daggett A, Logemann J, Rademaker A, et al. Laryngeal penetration during deglutition in normal subjects of various ages. Dysphagia 2006; 21: 270-274.

22 Robbins J, Coyle J, Rosenbek J, et al. Differentiation of normal and abnormal airway protection during swallowing using the penetration-aspiration scale. Dysphagia 1999; 14: 228-232.

23 Martin BJ, Logemann JA, Shaker R, et al. Coordination between respiration and swallowing: respiratory phase relationships and temporal integration. J Appl Physiol (1985) 1994; 76: 714-723.

24 Allen JE, White CJ, Leonard RJ, et al. Prevalence of penetration and aspiration on videofluoroscopy in normal individuals without dysphagia. Otolaryngol Head Neck Surg 2010; 142: 208-213. 
Mulheren RW, Azola AM, Kwiatkowski S, et al. Swallowing changes in community-dwelling older adults. Dysphagia 2018; 33: 848-856.

Garand KLF, Hill EG, Amella E, et al. Bolus airway invasion observed during videofluoroscopy in healthy, non-dysphagic community-dwelling adults. Ann Otol Rhinol Laryngol 2019; 128: 426-432.

Cassiani RA, Santos CM, Baddini-Martinez J, et al. Oral and pharyngeal bolus transit in patients with chronic obstructive pulmonary disease. Int J Chron Obstruct Pulmon Dis 2015; 10: 489-496.

de Deus Chaves R, Chiarion Sassi F, Davison Mangilli L, et al. Swallowing transit times and valleculae residue in stable chronic obstructive pulmonary disease. BMC Pulm Med 2014; 14: 62.

Macri MRB, Marques JM, Santos RS, et al. Clinical and fiberoptic endoscopic assessment of swallowing in patients with chronic obstructive pulmonary disease. Int Arch Otorhinolaryngol 2013; 17: 274-278.

Connors AF Jr, Dawson NV, Thomas C, et al. Outcomes following acute exacerbation of severe chronic obstructive lung disease. The SUPPORT investigators (Study to Understand Prognoses and Preferences for Outcomes and Risks of Treatments). Am J Respir Crit Care Med 1996; 154: 959-967.

Papi A, Bellettato CM, Braccioni F, et al. Infections and airway inflammation in chronic obstructive pulmonary disease severe exacerbations. Am J Respir Crit Care Med 2006; 173: 1114-1121.

Singh B. Impaired swallow in COPD. Respirology 2011; 16: 185-186.

Pragman AA, Lyu T, Baller JA, et al. The lung tissue microbiota of mild and moderate chronic obstructive pulmonary disease. Microbiome 2018; 6: 7.

Clayton NA, Carnaby-Mann GD, Peters MJ, et al. The effect of chronic obstructive pulmonary disease on laryngopharyngeal sensitivity. Ear Nose Throat J 2012; 91: 370-382.

Kaji A, Hashimoto Y, Kobayashi Y, et al. Sarcopenia is associated with tongue pressure in older patients with type 2 diabetes: a cross-sectional study of the KAMOGAWA-DM cohort study. Geriatr Gerontol Int 2019; 19: 153-158. Borders JC, Fink D, Levitt JE, et al. Relationship between laryngeal sensation, length of intubation, and aspiration in patients with acute respiratory failure. Dysphagia 2019; 34: 521-528.

Terpenning MS, Taylor GW, Lopatin DE, et al. Aspiration pneumonia: dental and oral risk factors in an older veteran population. J Am Geriatr Soc 2001; 49: 557-563.

Chalitsios CV, Shaw DE, McKeever TM. A retrospective database study of oral corticosteroid and bisphosphonate prescribing patterns in England. NPJ Prim Care Respir Med 2020; 30: 5.

Franssen FM, Spruit MA, Wouters EFM. Determinants of polypharmacy and compliance with GOLD guidelines in patients with chronic obstructive pulmonary disease. Int J Chron Obstruct Pulmon Dis 2011; 6: 493-501.

Martin-Harris B, McFarland D, Hill EG, et al. Respiratory-swallow training in patients with head and neck cancer. Arch Phys Med Rehabil 2015; 96: 885-893. 